\title{
Utility of trigger point injection as an adjunct to physical therapy in men with chronic prostatitis/chronic pelvic pain syndrome
}

\author{
Nicholas N. Tadros, Anup B. Shah, Daniel A. Shoskes \\ Department of Urology, Glickman Urologic and Kidney Institute, Cleveland Clinic, Cleveland, OH, USA \\ Contributions: (I) Conception and design: DA Shoskes; (II) Administrative support: DA Shoskes, AB Shah, NN Tadros; (III) Provision of study \\ materials or patients: DA Shoskes; (IV) Collection and assembly of data: All authors; (V) Data analysis and interpretation: All authors; (VI) \\ Manuscript writing: All authors; (VII) Final approval of manuscript: All authors. \\ Correspondence to: Daniel A. Shoskes, MD. Department of Urology, Glickman Urologic and Kidney Institute, Cleveland Clinic, 9500 Euclid Avenue, \\ Q10-1, Cleveland, OH 44195, USA. Email: dshoskes@gmail.com.
}

Background: Chronic prostatitis/chronic pelvic pain syndrome (CP/CPPS) is often associated with pelvic floor muscle spasm. While pelvic floor physical therapy (PFPT) is effective, some men are unable to resolve their symptoms and have residual trigger points (TPs). TP injection has been used for treatment in several neuromuscular pain syndromes. The objective of this study was to examine the efficacy and side effects of TP injection in men with CP/CPPS and pelvic floor spasm refractory to PT.

Methods: Using an IRB approved Men's Health Registry we reviewed the records of all men with a diagnosis of CP/CPPS who received at least 1 TP injection. Patients were phenotyped with UPOINT (all had the "T" domain for tenderness of muscle) and symptoms measured with the NIH Chronic Prostatitis Symptom Index (CPSI). Response was measured by a 5-point Global Response Assessment (GRA) and change in CPSI (paired $t$-test). For pelvic TPs, a pudendal block was done in lithotomy position and then each TP was identified transrectally by palpation. A nerve block needle was passed through the perineum into the TP confirmed by palpation. Between $0.5-1 \mathrm{cc}$ was injected into each TP of a local anesthetic mixture (30:70 of $2 \%$ lidocaine and $0.25 \%$ bupivacaine). For anterior TPs, an ultrasound guided ilioinguinal block was done first and then each TP injected by direct palpation through the abdominal skin. Men were offered up to three sets of injections separated by 6 weeks each.

Results: We identified 37 patients who had a total of 68 procedures. Three men had no follow-up after their first injection and were included for side effects but not included for outcome. The indication was failure to progress on PT in 33, recurrent symptoms in 1 and refusal to do PT in 3. Mean age was 43.7 years (range 21-70 years) and median UPOINT domains was 3 (range 1-5). Initial CPSI was pain $13.7 \pm 3.4$, urinary $5.3 \pm 2.2$, quality of life $9.8 \pm 2.1$ and total $28.8 \pm 6.0$. 16 men had 1 injection, 11 had 2 and 10 had 3. All had pelvic TPs injected and 9 also had anterior TPs. By GRA, 12 had significant improvement (35.3\%), 10 had some improvement (29.4\%), 11 had no change $(32.3 \%)$ and 1 was worse $(2.9 \%)$. Mean CPSI dropped from $28.8 \pm 6.0$ to $21.8 \pm 7.2$ (P<0.0001). 18 men had a drop of 6 or more points in CPSI $(53 \%)$. Of note, none of 3 men who were noncompliant with PT had benefit. 3 men had temporary numbness in the lateral thigh after the injection (4.4\%) and 1 had difficulty weight bearing on 1 leg for about 30 minutes.

Conclusions: TP injection in CP/CPPS patients as an adjunct to PT is well tolerated and leads to symptom improvement in about half. Durability and long term results are yet to be determined.

Keywords: Chronic prostatitis (CP); chronic pelvic pain; pelvic floor physical therapy (PFPT); trigger point (TP)

Submitted Apr 25, 2017. Accepted for publication May 08, 2017.

doi: $10.21037 /$ tau.2017.05.36

View this article at: http://dx.doi.org/10.21037/tau.2017.05.36 


\section{Introduction}

Chronic prostatitis/chronic pelvic pain syndrome (CP/ CPPS) remains a challenging condition to diagnose and treat. The creation of a syndrome based diagnostic classification by the National Institutes of Health (NIH) (1) has allowed for the development of a more focused approach to therapy based on symptoms without assuming etiology. Multimodal therapy based on the patient's phenotype has further allowed practitioners to better select patients with CP/CPPS that may respond to specific therapies.

Up to $85 \%$ of men with chronic pelvic pain may have pelvic floor tenderness (2) and these areas of tenderness reproduce the patient's pain with palpation in many cases (3). For these patients, the first line treatment is usually pelvic floor physical therapy (PFPT). Even in patients who have failed other therapies, PFPT has been shown to improve symptoms in up to $72 \%$ of these patients (4). Finding a therapist skilled in PFPT can be challenging and influence outcome (5). Unfortunately, PFPT is not a panacea and patients can fail or reach a plateau where they have no further improvement in their symptoms despite ongoing physical therapy. Trigger point (TP) injection is a standard therapy in other pain conditions with TPs and has been previously described in women with chronic pelvic pain with good results (6) and in at least one randomized trial it was equivalent to physical therapy in this population (7).

There is a paucity of literature in men with pelvic floor muscle spasm treated with TP injections (8), either as up front or adjunctive therapy. In this study we performed a retrospective review of all our male patients with pelvic floor spasm who underwent pelvic floor TP injection therapy to assess for improvement in pelvic pain and side effects.

\section{Methods}

Using an IRB approved (IRB\# 10-1047) Men's Health Registry we reviewed the records of all men with a diagnosis of CP/CPPS who received at least $1 \mathrm{TP}$ injection between April 2015 and January 2017. Three patients did not return and follow up was at least three months for the rest. Using the UPOINT phenotype classification system (9), all patients had a positive "T" domain for tenderness of pelvic floor muscles. Symptom severity was measured with the NIH Chronic Prostatitis Symptom Index (CPSI). Response to treatment was measured by a 5 -point Global Response
Assessment (GRA) and change in CPSI at each visit. All pelvic floor TP injections were performed by a single urologist (DS) with experience in the technique. The patient was first placed in the lithotomy position and a pudendal block was performed. Each TP was identified transrectally by palpation. A flexible 6 inch, 25 gauge needle (Havels ${ }^{\circledR}$, Cincinnati, Ohio) was passed through the perineum and into the TP as confirmed by palpation. Between $0.5-1 \mathrm{cc}$ of a local anesthetic mixture (30:70 of $2 \%$ lidocaine and $0.25 \%$ bupivacaine buffered with $10 \%$ sodium bicarbonate) was injected into each TP (Figure 1). For anterior TPs, an ultrasound guided ilioinguinal block was performed first and then each TP injected after identification via direct palpation through the abdominal skin. Men were offered up to three sets of injections separated by 6 weeks each.

Using Prism for Mac (La Jolla, CA, USA), paired $t$-tests were used for all statistical analysis and significance set at $\mathrm{P}<0.05$.

\section{Results}

Thirty-seven patients underwent a total of 68 procedures. Immediate complication data was available for all patients but follow up data was available for all but three patients. The average patient age was 43.7 (range 21-70) years. Median number of positive UPOINT domains was 3 (range 1-5). Initial CPSI scores were $13.7 \pm 3.4$ for pain, $5.3 \pm 2.2$ for urinary, and $9.8 \pm 2.1$ for quality of life. Total CPSI was $28.8 \pm 6.0$. Sixteen patients had one TP injection session, 11 had two sessions and 10 had three sessions. Nine patients received an anterior ilioinguinal block in addition to the TP injection(s). Indications for the procedures included noncompliance with PFPT [3], recurrent pain after PFPT [1] and failure to progress with symptom improvement on PFPT [33].

After treatment, total CPSI dropped to $21.8 \pm 7.2$ $(\mathrm{P}<0.0001)$. Eighteen men $(53 \%)$ had a drop of 6 or more points in their CPSI. By GRA, 12 patients (35.3\%) had significant improvement, ten $(29.4 \%)$ had some improvement, and $11(32.3 \%)$ had no change. One patient $(2.9 \%)$ was worse. Interestingly, none of 3 men who were noncompliant with physical therapy had a benefit from the TP injections.

Three men $(4.4 \%)$ had temporary numbness in the lateral thigh after the injection and one had difficulty weight bearing on one leg for about 30 minutes following the injection. 


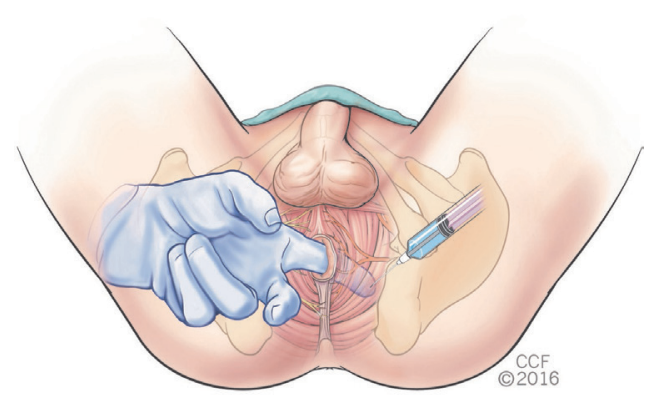

Figure 1 Transperineal injection of pelvic floor muscles with transrectal palpation of the trigger points

\section{Discussion}

Our study evaluated the effectiveness of TP injections in 37 patients with pelvic floor muscle spasm who failed to improve with pelvic floor physical therapy alone. 53\% of men saw a significant improvement in symptoms as measured by a minimum 6-point drop in their CPSI. The treatment was safe and we observed only minimal immediate complications that were of a short duration. Interestingly, there was no improvement in the three patients who were noncompliant with their PFPT. This emphasizes that the injection alone has a temporary effect and must be supplemented by continued stretching and muscle manipulation.

Men with recalcitrant CPPS often have undiagnosed pelvic floor spasm as a contributing cause. Pelvic floor physical therapy is the mainstay of treatment for pelvic floor muscle spasm but even in the best of hands does not improve all patients. In a study of 384 men with CPPS and 121 controls, pelvic floor tenderness was noted in $51 \%$ of the men with CPPS but in only $7 \%$ of the controls (2). Treating this pelvic floor spasm can yield significant improvement in patients' symptoms. In a study of 113 patients who had manual TP release, the patients saw a decrease in their average of visual analog pain scores from 7.5 to 4 after 6 months of treatment (10). There are still however a substantial number of patients who don't improve with physical therapy alone and treatment options for these patients has so far been limited. One option is medical therapy, which has been used with varying success depending on the drug. Neuromodulators such as amitriptyline and gabapentin have the potential to treat neuropathic pain and muscle spasm. A placebo controlled trial of amitriptyline in 50 patients showed an improvement in symptoms as measured by the O'Leary-Sant Interstitial Cystitis Score, especially in the domains of pain and urinary frequency (11). Gabapentin has been well studied as a treatment for neuropathic pain, but only small studies have investigated the medication as a treatment for CPPS. Sasaki et al. looked at 21 patients with refractory genitourinary pain (of all types, not just CPPS) who were treated with gabapentin. Ten patients had improvement in their pain, but $19 \%$ of their patients dropped out of the study due to side effects (12). Furthermore, stimulation of sacral nerves via an acupuncture technique has shown benefit by reducing CPSI in patients with CPPS in two separate studies $(13,14)$. Chen et al. notes a reduction in CPSI of $50 \%$ in 11 of 12 patients undergoing a 6-session acupuncture therapy with a median follow up time of 33 weeks. Kucuk et al. designed a prospective trial assessing medical (levofloxacin and ibuprofen) versus an acupuncture technique where the authors found that acupuncture reduced CPSI in $89 \%$ of patients by greater than $50 \%$, and the acupuncture group had a significantly larger drop in CPSI-pain sub score. Another potential treatment for refractory CPPS is extracorporeal shock wave therapy. Fifty-five patients (41 with complete data) refractory to at least three previous treatment modalities were treated with extracorporeal shock wave therapy once a week for one month. The mean CPSI improved 8.2 points for a $30 \%$ improvement in score with significant improvement seen at the two week point and remaining stable for 12 months (15).

The exact mechanism of TP injection benefit is still unknown. There is some evidence that the benefit may be due to the insertion of the needle into the muscle ("dry needling”) and not necessarily the substance injected $(16,17)$. Given the low incidence of complications, we have not tried omitting the local anesthetic for our procedure but realize that beneficial effects may come from the needle itself.

Our study was limited by the small number of patients and the short follow up. Even so this is the largest study to date on the utility of TP injections in men who have failed pelvic floor physical therapy. Further studies are needed to evaluate the long-term durability of this treatment.

\section{Conclusions}

TP injection in CP/CPPS patients when used as an adjunct to physical therapy is well tolerated and leads to symptom improvement in over half of these patients who have failed to progress with pelvic floor physical therapy. Durability 
and long term results are yet to be determined.

\section{Acknowledgements}

None.

\section{Footnote}

Conflicts of Interest: The authors have no conflicts of interest to declare.

Ethical Statement: This study was approved by the Cleveland Clinic IRB \#10-1047.

\section{References}

1. Krieger JN, Nyberg L Jr, Nickel JC. NIH consensus definition and classification of prostatitis. JAMA 1999;282:236-7.

2. Shoskes DA, Berger R, Elmi A, et al. Muscle tenderness in men with chronic prostatitis/chronic pelvic pain syndrome: the chronic prostatitis cohort study. J Urol 2008;179:556-60.

3. Anderson RU, Sawyer T, Wise D, et al. Painful myofascial trigger points and pain sites in men with chronic prostatitis/chronic pelvic pain syndrome. J Urol 2009;182:2753-8.

4. Anderson RU, Wise D, Sawyer T, et al. Integration of myofascial trigger point release and paradoxical relaxation training treatment of chronic pelvic pain in men. J Urol 2005;174:155-60.

5. Polackwich AS, Li J, Shoskes DA. Patients with pelvic floor muscle spasm have a superior response to pelvic floor physical therapy at specialized centers. J Urol 2015;194:1002-6.

6. Langford CF, Udvari Nagy S, Ghoniem GM. Levator ani trigger point injections: An underutilized treatment for chronic pelvic pain. Neurourol Urodyn 2007;26:59-62.

7. Zoorob D, South M, Karram M, et al. A pilot randomized trial of levator injections versus physical therapy for

Cite this article as: Tadros NN, Shah AB, Shoskes DA. Utility of trigger point injection as an adjunct to physical therapy in men with chronic prostatitis/chronic pelvic pain syndrome. Transl Androl Urol 2017;6(3):534-537. doi: 10.21037/ tau.2017.05.36 treatment of pelvic floor myalgia and sexual pain. Int Urogynecol J 2015;26:845-52.

8. Moldwin RM, Fariello JY. Myofascial trigger points of the pelvic floor: associations with urological pain syndromes and treatment strategies including injection therapy. Curr Urol Rep 2013;14:409-17.

9. Shoskes DA, Nickel JC, Dolinga R, et al. Clinical phenotyping of patients with chronic prostatitis/chronic pelvic pain syndrome and correlation with symptom severity. Urology 2009;73:538-42.

10. Anderson R, Wise D, Sawyer T, et al. Safety and effectiveness of an internal pelvic myofascial trigger point wand for urologic chronic pelvic pain syndrome. Clin J Pain 2011;27:764-8.

11. van Ophoven A, Pokupic S, Heinecke A, et al. A prospective, randomized, placebo controlled, doubleblind study of amitriptyline for the treatment of interstitial cystitis. J Urol 2004;172:533-6.

12. Sasaki K, Smith CP, Chuang YC, et al. Chancellor MB. Oral gabapentin (neurontin) treatment of refractory genitourinary tract pain. Tech Urol 2001;7:47-9.

13. Chen R, Nickel JC. Acupuncture ameliorates symptoms in men with chronic prostatitis/chronic pelvic pain syndrome. Urology 2003;61:1156-9; discussion 9.

14. Kucuk EV, Suceken FY, Bindayi A, et al. Effectiveness of acupuncture on chronic prostatitis-chronic pelvic pain syndrome category IIIB patients: a prospective, randomized, nonblinded, clinical trial. Urology 2015;85:636-40.

15. Al Edwan GM, Muheilan MM, Atta ON. Long term efficacy of extracorporeal shock wave therapy [ESWT] for treatment of refractory chronic abacterial prostatitis. Ann Med Surg (Lond) 2017;14:12-7.

16. Cummings TM, White AR. Needling therapies in the management of myofascial trigger point pain: a systematic review. Arch Phys Med Rehabil 2001;82:986-92.

17. Ay S, Evcik D, Tur BS. Comparison of injection methods in myofascial pain syndrome: a randomized controlled trial. Clin Rheumatol 2010;29:19-23. 